\title{
Partial Trisomy 13q/Monosomy 3p Resulting from a Paternal Reciprocal 3p;13q Translocation in a Boy with Facial Dysmorphism and Hypertrophic Cardiomyopathy
}

\author{
Monica Martin-de Saro ${ }^{a}$ Zyndia Compean $^{b}$ Karina Aguilar \\ Luz María González-Huertac Lautaro Plaza-Benhumead Olga Messina-Baasc \\ Sergio Alberto Cuevas-Covarrubiass ${ }^{c}$

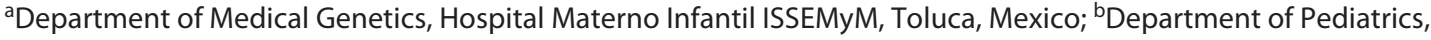

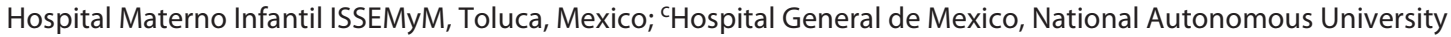

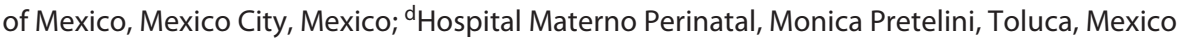

\section{Established Facts}

- Individuals with $3 p$ deletion show a great clinical variability, patients with partial trisomy $13 q$ usually have a phenotype resembling complete trisomy 13.

- Partial trisomy 13 regularly presents with heart defects.

\section{Novel Insights}

- We report a type of cardiomyopathy not previously reported.

- Few cases have been reported with chromosome imbalance due to a p3;q13 translocation.

- This is the first case with a $3 p$ deletion and $13 q$ duplication

\section{Keywords}

Trisomy $13 q \cdot$ Monosomy $3 p \cdot$ Translocation

\section{Abstract}

Individuals with $3 p$ deletion show a great clinical variability. Apparently, a 1.5-Mb terminal deletion, including the CRBN and CNTN4 genes, is sufficient to cause this syndrome. Partial trisomy $13 q$ is a rare chromosomal abnormality with a vari- able phenotypic expression, but in most cases, patients have a phenotype resembling complete trisomy 13 . The aim of the present study is to describe a 9-month-old Mexican male patient with $3 p$ deletion/13q duplication and a novel clinical finding. He presented with facial dysmorphism and multiple congenital alterations. Echocardiogram revealed cardiac insufficiency with hypertrophic cardiomyopathy and pulmonary hypertension, not previously reported. Karyotype from the patient and his father were $46, X Y, \operatorname{add}(3)(p 26)$ and
Correspondence to:

Sergio Alberto Cuevas-Covarrubiass, sergiocuevasunam @ gmail.com 
$46, X Y, t(3 ; 13)$, respectively. Microarray assay of the proband exhibited an approximately 2.6-Mb loss at terminal 3p26.3 and a 27.7-Mb gain of the long arm in terminal chromosome 13 at q31.1q34. A chromosomal imbalance with a partial trisomy $13 q 31.1 q 34$ and monosomy $3 p 26.3$ of paternal origin were detected. Microarray assay of both parents were normal. The proband has a cardiomyopathy not previously reported. These data enrich the spectrum of clinical manifestations in $3 p$ deletion/3q duplication chromosomopathy.

(c) 2021 S. Karger AG, Basel

\section{Introduction}

Chromosomal alterations that result in genomic imbalances are a major cause of congenital and developmental anomalies [Liu et al., 2016]. 3p deletion syndrome (OMIM 613792) is a contiguous gene syndrome associated with intellectual disability, developmental delay, intrauterine growth restriction, facial dysmorphism, skeletal abnormalities and other defects such as hypogenitalia, atrioventricular septal defects, hiatal hernia, optic atrophy, and polycystic renal dysplasia [Chen et al., 2012b; Moghadasi et al., 2014]. The spectrum of defects depends upon the overall size of the deleted segment [Kaur and Khetarpal, 2013]. The phenotype of individuals with $3 p$ deletions varies from normal to severe [Cuoco et al., 2011]. It has been suggested that a $1.5-\mathrm{Mb}$ minimal terminal deletion including the genes CRBN and CNTN4 in chromosome 3 is enough to cause this syndrome [Kaur and Khetarpal, 2013].

Partial trisomy $13 \mathrm{q}$ is a rare chromosomal abnormality with a variable phenotypic expression. It may result from parental reciprocal translocations and pericentric or paracentric inversions. Partial trisomy $13 \mathrm{q}$ has been shown to have a distinctive and common phenotype resembling that of complete trisomy 13. Common phenotypic features described for partial trisomy $13 \mathrm{q}$ are craniofacial dysmorphism, short neck, hemangioma, hexadactyly, urinary tract/kidney anomalies, umbilical/ inguinal hernia, intrauterine growth retardation, and oligohydramnios. Other phenotypic features in child and adult patients are psychomotor retardation, hypoacusia, hypochromic anemia, splenomegaly, ocular anomalies, convulsions, and fatty acid disturbances [Machado et al., 2010; Douglas et al., 2017].

This paper aims to describe a case with partial monosomy $3 p$ and partial trisomy $13 q$ with novel clinical data.

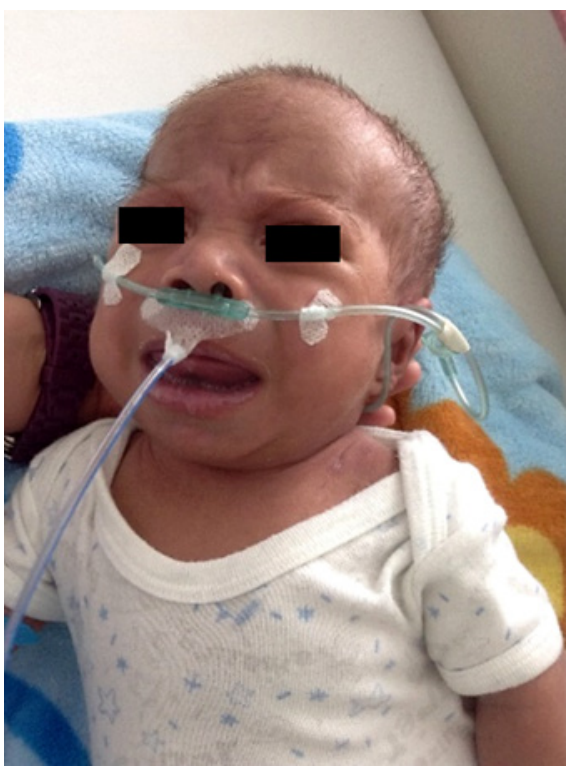

Fig. 1.Patient at 3 months of age showing anteverted nares.

\section{Case Presentation}

The proband, a 9-month-old male child, is the fourth child of young nonconsanguineous and healthy parents. No family history of congenital malformations was recorded. The child was born at 35 weeks after an uneventful pregnancy. At birth, he had a weight of $3,100 \mathrm{~g}$ (10th-25th centile), length of $50 \mathrm{~cm}$ (50th centile), occipitofrontal head circumference (OFC) of $33 \mathrm{~cm}$ (50th centile), and Apgar score of 9 at $5 \mathrm{~min}$. At the age of 2 months, he was referred to the hospital for hepatosplenomegaly, cholestasis, and facial dysmorphism.

On physical examination, at the age of 4 months, his weight was $4.2 \mathrm{~kg}$ ( $<3 \mathrm{rd}$ centile), length $53 \mathrm{~cm}(<3 \mathrm{rd}$ centile), and OFC $40 \mathrm{~cm}$ (3rd centile), he presented with upslanting palpebral fissures with long eyelashes, anteverted nares, high and narrow palate, heart murmur, umbilic hernia, and bilateral inguinal hernia with right cryptorchidism (Fig. 1). During hospitalization, he had cardiac insufficiency; an echocardiogram revealed hypertrophic cardiomyopathy and pulmonary hypertension. He had a normal hepatic ultrasound and a brain CT with cortical atrophy. He also had gastroesophageal reflux and needed gastrostomy for feeding. At 9 months, he persisted with low weight $(5.6 \mathrm{~kg}$; $3 \mathrm{rd}$ centile) and a low length $(59 \mathrm{~cm} ;<3$ rd centile).

\section{Materials and Methods}

Chromosome analysis was performed by routine GTG-banding at a band resolution of approximately 500. Genomic DNA was extracted with TRIzol reagent (Life Technologies, Santa Clara, CA, USA). Array analysis was carried out using CytoScan HD array (Affymetrix, Santa Clara, CA, USA), and scanned with the Affymetrix GeneChip Scanner 30007 G. Data were analyzed using GTYPE (GeneChip Genotyping Analysis Software, v1.0.12) to detect copy number aberrations. 


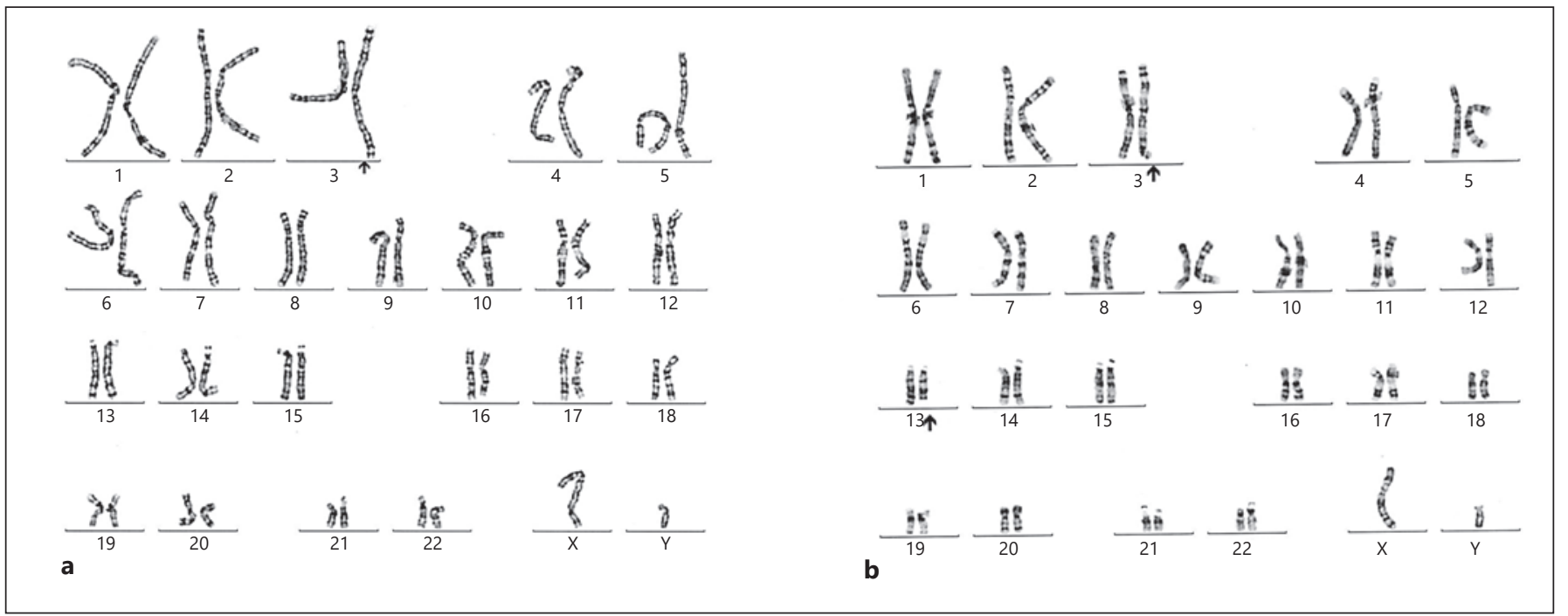

Fig. 2. Karyotypes. a Patient: 46,XY, add(3)(p26). b Father: 46,XY,t(3;13).
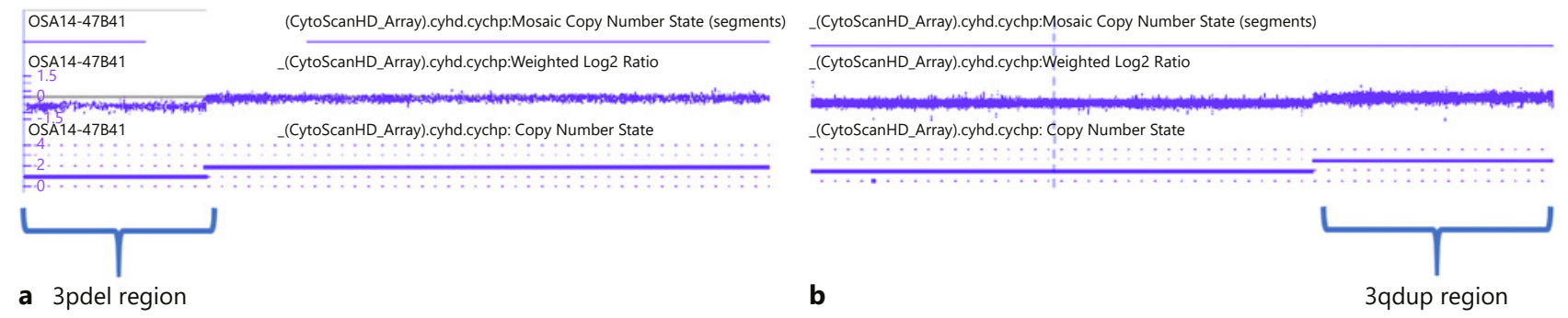

Fig. 3. a Microarray assay exhibiting a 2.6-Mb loss at terminal 3p26.3. b 27.7-Mb gain of the long arm in terminal chromosome 13 at q31.1q34. Genes involved are described in the text.

\section{Results}

Conventional cytogenetic analysis showed a karyotype of 46,XY,add(3)(p26) (Fig. 2a). Karyotype of the parents showed 46,XX (mother) and 46,XY,t $(3 ; 13)$ (father) (Fig. 2b). The oligonucleotide SNP assay had a $1.15-\mathrm{kb}$ resolution, and the result was: $\operatorname{arr}[\mathrm{hg} 19] 3 \mathrm{p} 26.3(61,891$ $2,312,694) \times 1,13 q 31.1 q 34\left(87,427,188 \_115,107,733\right) \times 3$, exhibiting an approximately $2.6-\mathrm{Mb}$ loss at terminal 3 p26.3 and a $27.7-\mathrm{Mb}$ gain of the long arm of terminal chromosome 13 at q31.1q34 (Fig. 3a, b). These data show a chromosomal imbalance with a partial trisomy $13 \mathrm{q} 31.1 \mathrm{q} 34$ and a monosomy $3 \mathrm{p} 26.3$ of paternal origin. Genes involved are shown in Table 1. The microarray analysis of both parents was normal.

$3 p$ deletion/13q duplication Syndrome

\section{Discussion and Conclusion}

The $3 p$ deletion syndrome is a rare contiguous gene syndrome caused by deletions with variable size in 3 p25pter; the deletion ranges from one to several megabases. There are no common breakpoints, and it mostly occurs de novo; only a few familial cases have been reported [Cuoco et al., 2011]. The minimal deletion region is thought to be $1.5 \mathrm{Mb}$ in size and includes the $C R B N$ and CNTN4 genes [Kashevarova et al., 2014]. To better define the genetic basis of $3 p$ deletion syndrome, a cohort of 14 patients with visible distal $3 p$ deletions has been studied by SNP array. Among the different haploinsufficient genes, a deletion involving CRBN and CNTN4 seems to be enough to produce the typical clinical features, while 
Table 1. Disease-causing genes

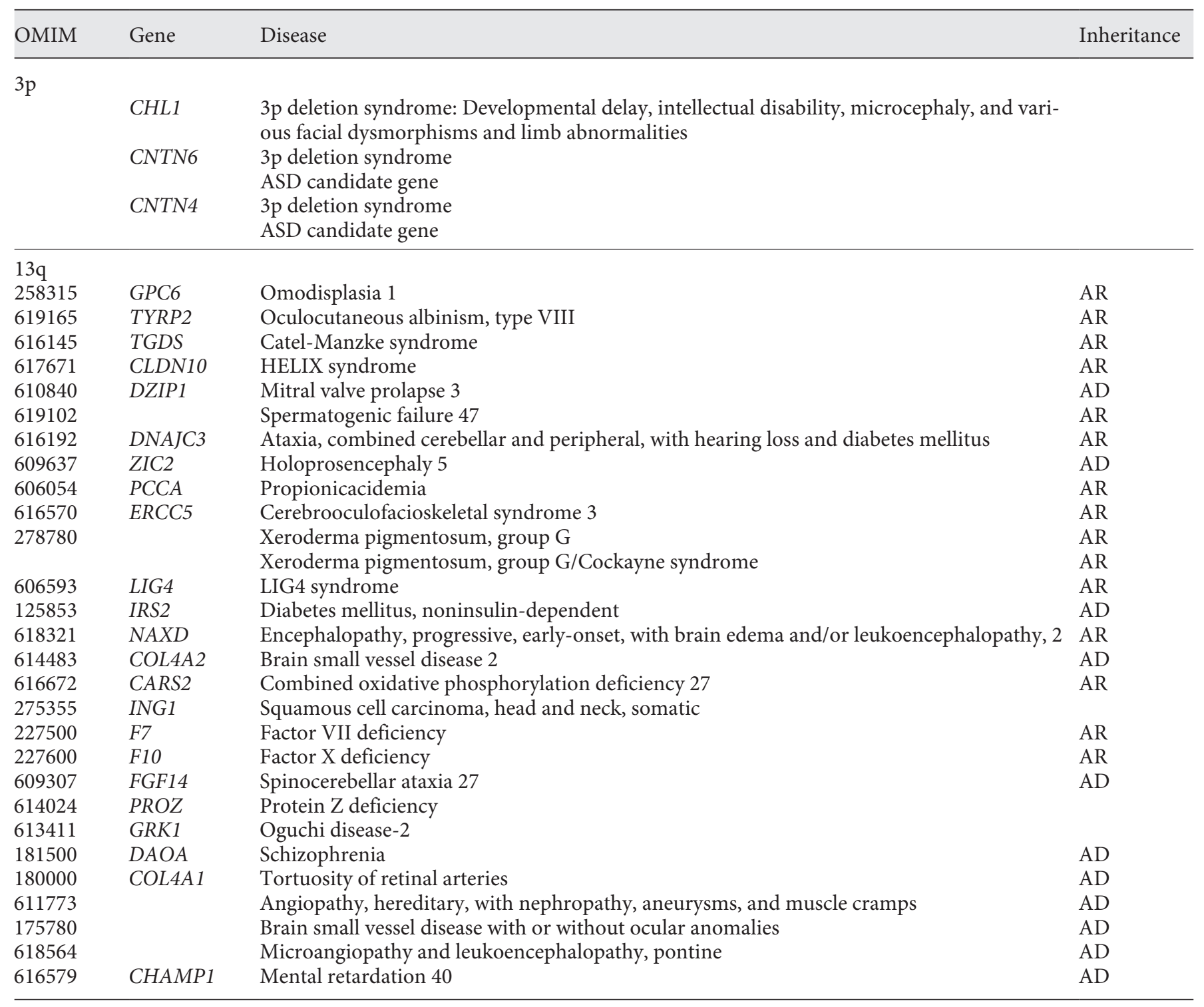

ASD, autism spectrum disorder; AD, autosomal dominant; AR, autosomal recessive. Reference, UCSC Genome Browser on Human Dec. 2013 (GRCh38/hg38). Assembly, https://genome.ucsc.edu/.

the $C H L 1$ gene has been suggested to contribute to mental development [Cuoco et al., 2011; Tassano et al., 2014].

To explain the variability in the phenotype from severe to normal, chromosomal non-penetrance and gene modification have been proposed [Sims et al., 2012].

In our case, the copy number loss involves CHL1, CNTN6, CNTN4 andCNTN4-AS2. CNTN4 mRNA transcript is a member of the immunoglobulin superfamily of neuronal cell adhesion molecules; it participates in axon growth, guidance, and fasciculation in the central nervous system (CNS). The CNTN4 disruption is associated with the $3 p$ deletion syndrome phenotype; knockouts of homologous neuronal adhesion molecules in mouse have resulted in viable mutants demonstrating morphological, neurological and behavioral abnormalities. This indicates an important role for CNTN4 in CNS development [Fernandez et al., 2004]. This gene may play a role in 3p deletion syndrome with low birth weight, microcephaly, trigonocephaly, hypertelorism, ear abnormalities, hypotonia, psychomotor and growth retardation, among others [Cervantes et al., 2014; Iype et al., 2015]. Our patient has microcephaly, psychomotor and growth retardation. 
Table 2. Comparative table between the proband and $3 p$ deletion and $13 q$ duplication syndrome

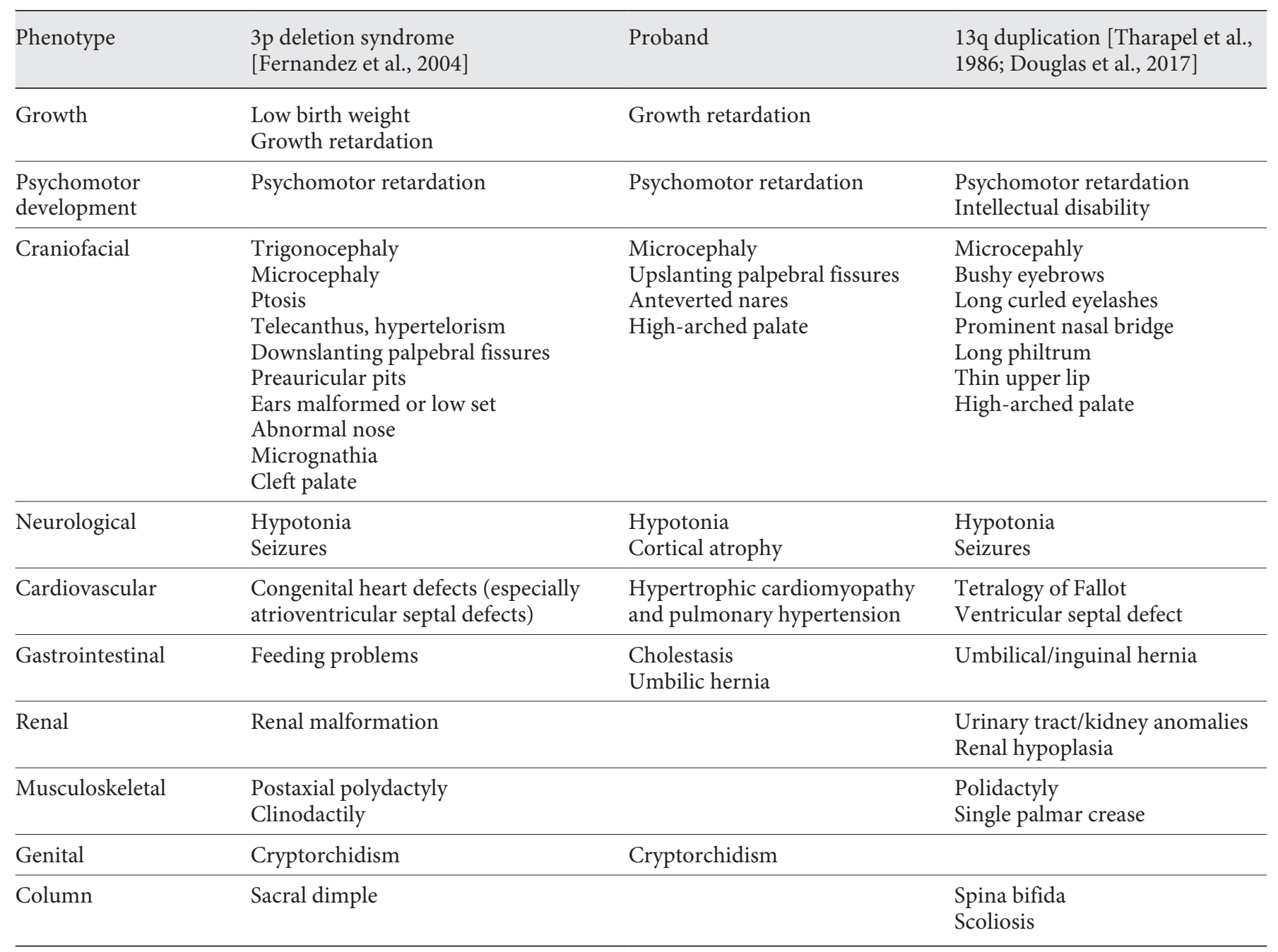

The region distal to the disrupted CNTN4 gene contains CNTN6 and CHL1; they are neuronal cell adhesion molecules that also contain Ig and FNIII domains. Analyses of CHL1 knockout mice have identified anomalies in axonal organization in various brain regions as well as subtle behavioral abnormalities in homozygous null animals [Fernandez et al., 2004].

In order to further investigate genotype-phenotype correlations in 3p deletion syndrome, Shuib et al. [2009] analyzed 14 patients with cytogenetically detectable deletions of 3p25 (including one patient with a normal phenotype) using Affymetrix 250K SNP microarrays. A candidate critical region for a congenital heart disease susceptibility gene was refined to approximately $200 \mathrm{~kb}$ and a candidate critical region for intellectual disability was mapped to an approximately $1-\mathrm{Mb}$ interval containing

$3 \mathrm{p}$ deletion/13q duplication Syndrome
SRGAP3. Other $3 \mathrm{p}$ neuro developmental genes including CHL1, CNTN4, LRRN1, and ITPR1 mapped outside the candidate critical interval. This current evidence suggests that SRGAP3 is the major determinant gene of intellectual disability in distal 3p deletions [Shuib et al., 2009].

CHL1 is a member of the L1 family of cell adhesion molecules, these proteins play an important role in building and functioning of the brain. Due to the fact that migration and synaptogenesis require cell to cell and cell to matrix interactions, it has been suggested that these molecules are associated with intellectual defects in patients with 3p deletion syndrome [Cuoco et al., 2011; Gandawijaya et al., 2021].

CNTN6 is a neuronal membrane protein that functions as a cell adhesion molecule and may contribute to the formation of axon connections in the developing ner- 
vous system. CNTN6, and other members of this protein family, have been identified as potential disease-causing genes in neurodevelopmental disorders. It has been suggested that these genes participate in pathways that are important for correct brain development. In mice, they are thought to participate in embryonic development and postnatal brain maturation. CNTN6 deficiency in mice causes profound motor coordination abnormalities and learning difficulties [Kashevarova et al., 2014].

There are previous reports of patients with $3 p$ deletion syndrome and different congenital cardiopathies; Chen et al. [2012a] reported coarctation of the aorta, patent ductus arteriosus, peripheral pulmonary stenosis, and atrial septal defect. The oligonucleotide-based array CGH revealed a $4.79-\mathrm{Mb}$ deletion of $3 \mathrm{p} 26.2 \rightarrow$ pter encompassing CHL1 and CNTN4, and a $19.56-\mathrm{Mb}$ duplication of $5 \mathrm{q} 34 \rightarrow$ qter encompassing MSX2, NKX2-5, and NSD1. The karyotype of the girl was $46, \mathrm{XX}, \operatorname{der}(3) \mathrm{t}(3 ; 5)$ (p26.2;q34)pat. The authors attribute the cardiopathy to the distal $5 \mathrm{q}$ duplication, not to the $3 \mathrm{p}$ deletion [Chen et al., 2012a]. Our patient had hypertrophic cardiomyopathy and pulmonary hypertension, not previously reported, maybe secondary to the $13 \mathrm{q}$ duplication.

Patients with distal $13 \mathrm{q}$ duplications typically show features overlapping those with full trisomy 13 [Helali et al., 1996]. Genomic rearrangements at chromosome 13q31.3q32.1 have been associated with multiple anomalies and a variable degree of mental disability [Tharapel et al., 1986; Brewer et al., 1999; Machado et al., 2010; Wei et al., 2012; Kannu et al., 2013].

Kannu et al. [2013] reviewed eight 13q duplication cases finding important phenotypic differences, 5 presented with facial dysmorphism, 4 of them had developmental delay, and only 1 had a nonspecified heart defect [Nikolis et al., 1991; Kannu et al., 2013].

Table 2 presents the symptoms between $3 p$ deletion syndrome, $13 \mathrm{q}$ duplication syndrome and our patient.

McCorquodale et al. [1980] reported a child with partial trisomy involving the proximal segment of the long arm of chromosome 13 derived from a paternal translocation (p3;q13). The final karyotype was $47, \mathrm{XY},+\operatorname{der}(13)$ $t(3,13)$ (q24;q14). This boy had a clover-leaf shape of the head, CNS malformations, small sacral myelomeningocele, developmental delay, glaucoma, low nasal bridge, snub nose, prominent philtrum, narrow and high palate, a short neck with increased skin folds in the nape, asymmetric chest, small penis, right testis not palpable and left testis in the left inguinal canal, hands showed ulnar loops, hyperextension at interphalangeal joints, prominent heels, and hypotrophic toe nails. Above all these manifes- tations, our patient also had developmental delay, high and narrow palate, and cryptorchidism.

In conclusion, we reported a case with $13 \mathrm{q}$ duplication and $3 p$ deletion in a patient with hypertrophic cardiomyopathy and pulmonary hypertension, not previously reported. The presence of heart defect previously described indicates that this data could be part of this syndrome. It is important to mention that besides the proband is not the first case reported with this type of translocation; however, it is the first patient with a $3 p$ deletion and $13 q$ duplication.

\section{Acknowledgement}

We thank the patient and his family for participating in this study.

\section{Statement of Ethics}

Both parents gave their written informed consent prior to the realization of the studies and authorized to publish the case including images. This study protocol was reviewed and approved by Comite de Etica en Investigacion and Comite de Investigacion of Hospital General de Mexico, approval number DI/17/310A/04/083.

\section{Conflict of Interest Statement}

The authors have no conflicts of interest to declare.

\section{FundingSources}

Programa de Posgrado en Ciencias Médicas, Odontológicas y de la Salud, Facultad de Medicina, UNAM. PAPIIT IN219419, DGAPA, Universidad Nacional Autonoma de México.

\section{Author Contributions}

Writing and original draft preparation: M.D.M., S.A.C., Z.C., K.A., L.M.G. O.M.B., and L.P.B. Critical review: M.D.M., K.A., and S.A.C. All authors analyzed and interpreted the data and approved the manuscript in its final form.

\section{References}

Brewer C, Holloway S, Zawalnyski P, Schinzel A, FitzPatrick D. A chromosomal duplication map of malformations: regions of suspected haplo- and triplo lethality-and tolerance of segmental aneuploidy-in humans. Am J Hum Genet. 1999;64(6):1702-8.

Cervantes A, García-Delgado C, FernándezRamírez F, Galaz-Montoya C, Morales-Jiménez $A B$, Nieto-Martínez $K$, et al. Trisomy 1q41-qter and monosomy 3p26.3-pter in a family with a translocation $(1 ; 3)$ : further delineation of the syndromes. BMC Med Genomics. 2014;7:55. 
Chen C, Lin S, Chen M, Su Y, Chern S, Liu Y, et al. Partial monosomy $3 p$ (3p26.2-->pter) and partial trisomy $5 \mathrm{q}$ ( $5 \mathrm{q} 34-->$ qter $)$ in a girl with coarctation of the aorta, congenital heart defects, short stature, microcephaly and developmental delay. Genet Couns. 2012a;23(3): 405-13.

Chen C, Su Y, Chen C, Su J, Chern S, Town D, et al. Pure partial monosomy 3p (3p25.3 $\rightarrow$ pter): prenatal diagnosis and array comparative genomic hybridization characterization. Taiwan J Obstet Gynecol. 2012b;51(3):435-9.

Cuoco C, Ronchetto P, Gimelli S, Béna F, Divizia MT, Lerone M, et al. Microarray based analysis of an inherited terminal 3p26.3 deletion, containing only the CHL1 gene, from a normal father to his two affected children. Orphanet J Rare Dis. 2011;6:12.

Douglas C, Smith SA, Rohena L. Novel case of paternal paracentric inversion causing partial trisomy 13 and review of the literature. Am J Med Genet A. 2017;173(6):1673-80.

Fernandez T, Morgan T, Davis N, Klin A, Morris A, Farhi A, et al. Disruption of Contactin 4 (CNTN4) Results in Developmental Delay and Other Features of 3p Deletion Syndrome. Am J Hum Genet. 2004;74(6):1286-93.

Gandawijaya J, Bamford RA, Burbach JPH, Oguro-Ando A. Cell Adhesion Molecules Involved in Neurodevelopmental Pathways Implicated in 3p-Deletion Syndrome and $\mathrm{Au}$ tism Spectrum Disorder. Front Cell Neurosci. 2020;14:611379.

Helali N, Iafolla A, Kahler S, Qumsiyeh M. A case of duplication of 13q32-->qter and deletion of 18 p11.32--> pter with mild phenotype: Patau syndrome and duplications of $13 \mathrm{q}$ revisited. J Med Genet. 1996;33(7):600-2.
Iype $T$, Alakbarzade $V$, Iype $M$, Singh $R$, Sreekantan-Nair A, Chioza BA, et al. A large Indian family with rearrangement of chromosome 4p16 and 3p26.3 and divergent clinical presentations. BMC Med Genet. 2015; 16:104.

Kannu P, Campos-Xavier AB, Hull D, Martinet D, Ballhausen D, Bonafé L. Post-axial polydactyly type A2, overgrowth and autistic traits associated with a chromosome 13q31.3 microduplication encompassing miR-17-92 and GPC5. Eur J Med Genet. 2013;56(8):4527.

Kashevarova AA, Nazarenko LP, Schultz-Pedersen S, Skryabin NA, Salyukova OA, Chechetkina NN, et al. Single gene microdeletions and microduplication of 3 p26.3 in three unrelated families: CNTN6 as a new candidate gene for intellectual disability. Mol Cytogenet. 2014; 7(1):97.

Kaur A, Khetarpal S. 3p Deletion Syndrome. Indian Pediatr. 2013;50(8):795-6.

Liu HY, Huang J, Li T, Wu D, Wang HD, Wang $\mathrm{Y}$, et al. Clinical and molecular cytogenetic analyses of four patients with imbalanced translocations. Mol Cytogenet. 2016;9:31.

Machado I, Heinrich J, Campanhol C, Rodrigues R, Oliveira F, Barini R, et al. Prenatal diagnosis of a partial trisomy 13q (q14-->qter): phenotype, cytogenetics and molecular characterization by spectral karyotyping and array comparative genomic hybridization. Genet Mol Res. 2010;9(1):441-8.

McCorquodale M, Erickson R, Robinson M, Roszczipka K. Kleeblattschädel anomaly and partial trisomy for chromosome 13 $(47, \mathrm{XY},+\operatorname{der}(13) \mathrm{t}(3,13)(\mathrm{q} 24 ; \mathrm{q} 14)$. Clin Genet. 1980;17(6):409-14.
Moghadasi S, van Haeringen A, Langendonck L, Gijsbers AC, Ruivenkamp CA. A terminal 3p26.3 deletion is not associated with dysmorphic features and intellectual disability in a four-generation family. Am J Med Genet A. 2014;164A(11):2863-8.

Nikolis J, Ivanović K, Diklić V. Partial trisomy 13q resulting from a paternal reciprocal Yq;13q translocation. J Med Genet. 1991;28(6):425-

Shuib S, McMullan D, Rattenberry E, Barber RM, Rahman F, Zatyka M, et al. Microarray based analysis of 3p25-p26 deletions (3p-syndrome). Am J Med Genet A. 2009;149A(10): 2099-105.

Sims K, Mazzaschi R, Payne E, Hayes I, Love D, George A. A Rare Chromosome 3 Imbalance and Its Clinical Implications. Case Rep Pediatr. 2012;2012:846564.

Tassano E, Biancheri R, Denegri L, Porta S, Novara $\mathrm{F}$, Zuffardi $\mathrm{O}$, et al. Heterozygous deletion of CHL1 gene: detailed array-CGH and clinical characterization of a new case and review of the literature. Eur J Med Genet. 2014; 57(11-12):626-9.

Tharapel SA, Lewandowski RC, Tharapel AT, Wilroy RS. Phenotype-karyotype correlation in patients trisomic for various segments of chromosome 13. J Med Genet. 1986;23(4): 310-5.

Wei Y, Gao X, Yan L, Xu F, Li P, Zhao Y. Prenatal diagnosis and postnatal follow up of partial trisomy $13 \mathrm{q}$ and partial monosomy 10p: a case report and review of the literature. Case Rep Genet. 2012;2012:821347. 